\title{
A simple game-theoretic approach to checkonly QVT Relations
}

\author{
Perdita Stevens \\ Laboratory for Foundations of Computer Science \\ School of Informatics \\ University of Edinburgh
}

December 2009

\begin{abstract}
The QVT Relations (QVT-R) transformation language allows the definition of bidirectional model transformations, which are required in cases where a two (or more) models must be kept consistent in the face of changes to either. A QVT-R transformation can be used either in checkonly mode, to determine whether a target model is consistent with a given source model, or in enforce mode, to change the target model. Although the most obvious semantic issues in the QVT standard concern the restoration of consistency, in fact even checkonly mode is not completely straightforward; this mode is the focus of this paper. We need to consider the overall structure of the transformation as given by when and where clauses, and the role of trace classes. In the standard, the semantics of QVT-R are given both directly, and by means of a translation to QVT Core, a language which is intended to be simpler. In this paper, we argue that there are irreconcilable differences between the intended semantics of QVT-R and those of QVT Core, so that the translation cannot be helpful. Treating QVT-R directly, we propose a simple game-theoretic semantics. We demonstrate its behaviour on examples and show how it can be used to compare QVT-R transformations. We demonstrate that consistent models may not possess a single trace model whose objects can be read as traceability links in either direction. We briefly discuss the effect of variations in the rules of the game, to elucidate some design choices available to the designers of the QVT-R language.
\end{abstract}

\section{Introduction}

Model-driven development (MDD) is widely agreed to be an important ingredient in the development of reliable, maintainable multi-platform software. The Object Management Group, OMG, is the industry's consensus-based standards body, so the standards it proposes for model-driven development are necessarily 
important. In the area of MDD, a key standard is Queries, Views and Transformations (QVT, [5]), a specification of three different languages for defining transformations between models, which may include defining a restricted view of a model which abstracts away from aspects of the model not relevant to a particular class of intended user. Rather disappointingly, however, the Queries, Views and Transformations languages have been slow to be adopted. Few tools are available for any of the languages: notably, it sometimes happens that even those tools which use "QVT" in their marketing literature do not actually provide any of the three QVT languages, but rather, provide a "QVTlike" language. In this paper we will consider QVT Relations (QVT-R), the language which best permits the high-level, declarative specification of bidirectional transformations. There have been two candidate implementations of this: Medini QVT ${ }^{1}$ and ModelMorf ${ }^{2}$. ModelMorf is the more faithful to [5], and will be discussed further in this article.

Why has the uptake of QVT been so low? Optimistically, we may point to the fact that, while the QVT standard has been under development for a long time, it has only recently been standardised. However, the same applied to other OMG standards, most notably UML, and did not prevent their adoption before finalisation. Lack of support for important engineering activities like testing and debugging may also play a role, but this does not explain why there do exist several tools each of which uses its own transformation language other than the OMG standard ones, and case studies of successful use of these tools. Perhaps a contributory factor is that, whereas the UML standard was developed following years of widespread use of various somewhat similar modelling languages, the model transformation arena is still far more sparsely populated. Therefore, how to define, or recognise, a good model transformation language for use on a particular problem is less well understood. We consider that the difficulty developers have in understanding the semantics of QVT may play a role, and we develop a game-theoretic semantics which we hope may be more accessible.

In this paper, we only consider transformations in checkonly mode. That is, we are interested in the case where a QVT-R transformation is presented with two or more models, and the transformation engine must return true if the models are consistent according to the definition of consistency embodied in the transformation, or false otherwise. Perhaps surprisingly, it turns out that this already raises some interesting issues.

Related work This is an extended version of a paper [11] presented at the International Conference on Model Transformations in June 2009. As well as giving more formal details, this paper adds two new sections: Section 6, which applies the new semantics to a family of examples and compares the results with those from ModelMorf, and Section 7, which shows how the game semantics can be exploited to prove results about the (anti-)equivalence of checkonly QVT-R

\footnotetext{
${ }^{1}$ http://projects.ikv.de/qvt/, version 1.6 .0 current at time of writing

${ }^{2}$ http://www.tcs-trddc.com/ModelMorf/index.htm, but this page is (once again) not available at time of writing. This is a known problem [6]
} 
transformations. We also discuss the nature and role of trace objects in QVT-R more thoroughly. The papers follow on from earlier work by the present author, [10], in which questions answered here, specifically the role of relation invocation in when and where clauses (relation definition applied to particular arguments), were left open. Discussion of the foundations of, and range of approaches to, bidirectionality, not specific to QVT, are presented in [9] and [8] respectively.

Greenyer and Kindler [3] presented at MODELS 2007 a discussion of the relationship between QVT Core and Triple Graph Grammars, together with an outline of a translation from QVT Core to TGGs. Romeikat and others [7] translated QVT-R transformations to QVT Operational. Garcia [1] formalised aspects of QVT-R in Alloy, permitting certain well-formedness errors to be detected.

Formal games have been widely used in computer science; the most relevant strand for this paper is surveyed in [12]. In modelling, the GUIDE tool [13] uses games to support design exploration and verification.

\section{Background}

QVT Relations A QVT-R transformation is structured as a number of relations, connected by referencing one another in when and where clauses. The idea is that an individual relation constrains a tuple of models in a rather simple, local, way, by matching patterns rooted at model elements of particular kinds. The power, and the complexity, of the transformation comes from the way in which relations are connected. A relation may also have a when clause and/or a where clause. In these clauses, other relations are invoked with particular roots for their own patterns to be matched. In this way, global constraints on the models being compared can be constructed from a web of local constraints. The allowed dependencies between the choices made of values for variables in a typical implementation, the order in which these choices are made - are such that the when functions as a kind of pre-condition; the where clause imposes further constraint on the values chosen during the relation to which it is attached (it is, in a way, a post-condition).

The reader is referred to [5] for details: the relevant sections are Chapter 7 and Appendix B. A key point is that the truth of a relation is defined using a logical formula which states that for every legal assignment of values to certain variables, there must exist an assignment of values to certain other variables, such that a given condition is satisfied.

Logic In logical terms, this is expressed as a "for all-there exists" formula; more precisely, such a formula is called a $\Pi_{2}$ formula, provided that the formula which follows these two quantifiers is itself quantifier-free.

The difficulty in QVT-R is that actually, the truth of a complete transformation is expressed by a much more complex formula. Appendix B only expresses the truth of an individual relation, but this is defined in terms of the truth of the relations which may appear in its when and where clauses, so that, in fact, the 
number of alternations between universal and existential quantifiers (the length of a forall-thereexists-forall-thereexists... formula which would be equivalent to a whole QVT-R transformation evaluating to true) is unbounded. For example, consider the well-known example of transformation between UML class diagrams and RDBMS schemas, in which packages correspond to schemas, classes to tables and attributes to columns. Looking at [5] p197, we see that ClassToTable invokes relation AttributeToColumn in its where clause. The invocation gives explicit values for the root variables of the patterns in AttributeToColumn, but even though those are fixed, the usual rule applies as regards the rest of the valid bindings to be found in AttributeToColumn. Thus, for each valid binding of one pattern in ClassToTable (and of the when variables), there must exist a valid binding of the other pattern in ClassToTable, such that for each valid binding of the remaining variables of one pattern in AttributeToColumn (and of the when variables, except that in this case there are none), there exists a valid binding of the remaining variables of the other pattern in AttributeToColumn. ${ }^{3}$ Note that, if there was more than one choice for the second binding in ClassToTable, it is entirely possible that it turns out that only one of these choices satisfies the rest of the condition, concerning the matching in AttributeToColumn: thus any evaluation, whether mental or by a tool, of ClassToTable has to be prepared either to consider both relations together, or to backtrack in the case that the first choice of binding made is not the best.

Therefore, while one might at first glance hope to be able to understand, and evaluate, the meaning of a QVT-R transformation by studying the relations individually, in fact, no such "local" evaluation is possible, because of the way the relations are connected.

Fortunately, similar situations arise throughout logic and computer science, and much work has been done on how to handle them. In particular, this is exactly the situation in which games have found to be a useful aid to developing intuition, as well as to formal reasoning.

Games There is a long history in logic of formulating the truth of a logical proposition as the existence of a winning strategy in a two-player game. For example, the formula $\forall x . \exists y \cdot y>x$ (where $x$ and $y$ are integers, say) can be turned into a game between two players. The player who is responsible for picking a value for $x$ is variously called $\forall$ belard, Player I, Spoiler, Refuter, depending on the community defining the game, while the player responsible for picking a value for $y$ is called $\exists$ louise, Player II, Duplicator or Verifier. We will go with Refuter and Verifier. Refuter's aim is, naturally, to refute the formula, while Verifier's aim is to verify it. In this game, Refuter has to pick a value for $x$, then Verifier has to pick a value for $y$. Verifier then wins this play of the game if $y>x$, while Refuter wins this play otherwise. This is an example of a two-player game of perfect information (that is, both players can

\footnotetext{
${ }^{3}$ Actually, the version in [5] is a little more complicated than this: AttributeToColumn invokes further relations in its where clause, and it is those which require the binding of remaining variables: but the point is the same.
} 
see everything about one another's moves). In fact, in this case, Verifier has a winning strategy for the game: that is, she has a way of winning the game in the face of whatever moves Refuter may choose. For example, she could decide always to obtain her value of $y$ by adding 42 to whatever value of $x$ is chosen by Refuter.

Formally speaking, we define a game as follows. Notice that our definition allows for the possibility of plays of a game being infinite, although it may happen that a particular game is defined in such a way that all plays are finite. We will use Player $P$, Player $\bar{P}$ to mean Verifier and Refuter in either order; that is, if Player $P$ is Verifier then Player $\bar{P}$ is Refuter, and vice versa.

Definition 1. A game $G$ is (Pos, Initial, moves, $\left.\lambda, W_{R}, W_{V}\right)$ where:

- Pos is a set of positions. We use $u, v, \ldots$ for positions.

- $\lambda:$ Pos $\rightarrow\{$ Verifier, Refuter $\}$ defines who moves from each position.

- Initial $\in$ Pos is the starting position: for purposes of this paper, $\lambda($ Initial $)=$ Refuter.

- moves $\subseteq$ Pos $\times$ Pos defines which moves are legal. A play is in the obvious way a finite or infinite sequence of positions, starting with Initial, where $p_{j+1} \in \operatorname{moves}\left(p_{j}\right)$ for each $j$. We write $p_{i j}$ for $p_{i} \ldots p_{j}$.

- $W_{R}, W_{V} \subseteq$ Pos $^{\omega}$ such that $W_{R} \cup W_{V}=$ Pos $^{\omega}$ are disjoint sets of infinite plays, and (for technical reasons) $W_{P}$ includes every infinite play $p$ such that there exists some $i$ such that for all $k>i, \lambda\left(p_{k}\right)=\bar{P}$.

Player $P$ wins a play $p$ if either $p=p_{0 n}$ and $\lambda\left(p_{n}\right)=\bar{P}$ and $\operatorname{moves}\left(p_{n}\right)=\emptyset$ (you win if your opponent can't go), or else $p$ is infinite and in $W_{P}$.

Then a strategy for such a game is, informally, a set of instructions for one player, telling the player how to move in response to any (legal) move of the opponent. A strategy may be deterministic - it tells the player exactly how to move - or non-deterministic - it gives a set of possible moves. In general, the move prescribed by the strategy may depend on the entire play so far. A strategy in which the moves prescribed only depend on the current position (not on the way in which the current position was reached) is called memoryless or history-free. More formally:

Definition 2. $A$ (nondeterministic) strategy $S$ for player $P$ is a partial function from finite plays pu with $\lambda(u)=P$ to sets of positions (singletons, for deterministic strategies), such that $S(p u) \subseteq$ moves $(u)$ (that is, a strategy may only prescribe legal moves). A play $q$ follows $S$ if whenever $p_{0 n}$ is a proper finite prefix of $q$ with $\lambda\left(p_{n}\right)=P$ then $p_{n+1} \in S\left(p_{0 n}\right)$. Thus an infinite play follows $S$ whenever every finite prefix of it does. It will be convenient to identify a strategy with the set of plays following the strategy and to write $p \in S$ for $p$ follows $S$. $S$ is a complete strategy for Player $P$ if whenever $p_{0 n} \in S$ and $\lambda\left(p_{n}\right)=P$ then $S\left(p_{0 n}\right) \neq \emptyset$. It is a winning strategy for $P$ if it is complete and every $p \in S$ is 
either finite and extensible or is won by $P$. It is history-free (or memoryless) if $S(p u)=S(q u)$ for any plays pu and qu with a common last position. A game is determined if one player has a winning strategy.

All the games we need to consider are determined by standard game theory [4]: in fact, one player or the other will have a memoryless deterministic winning strategy. In this simpler situation, and for games that have no infinite plays, a strategy for Player $P$ will simply be a partial function $S$ from positions $u$ that have $\lambda(u)=P$ to positions, such that $S(u) \in$ moves $(u)$, that is, the strategy only prescribes legal moves. For practical purposes, it will suffice to define the strategy at positions that are actually reachable by following the strategy (allowing, of course, for all possible choices by the opponent, Player $\bar{P}$ ).

Returning to our example of the logic game based on $\forall x . \exists y \cdot y>x$, let us point out that it is of course entirely possible that a player has more than one winning strategy. When a $\Pi_{2}$ formula is true, a Skolem function expresses a particular set of choices that constitute a winning strategy: given $x$, it returns the chosen $y$. Different Skolem functions may exist which justify the truth of the same formula. In the example above, one choice of Skolem function maps $x$ to $x+1$, another maps $x$ to $x+17$, another maps 1 to 23,2 to 4,3 also to 4 , and so on. Clearly the trace model in QVT has something in common with a Skolem function: it expresses a way in which parts of one structure may be mapped to "corresponding" parts of another. We do not yet have a game for QVT transformations that would enable us to make this notion of correspondence precise, however: we will return to the issue in Section 8.2.

Another family of examples, which may get us closer, comes from concurrency theory. Processes are modelled as labelled transition systems (LTSs), that is, an LTS is a set of states $S$ including a distinguished start state $i \in S$, a set of labels $L$, and a ternary relation $\rightarrow \subseteq S \times L \times S$ : we write $s \stackrel{a}{\rightarrow} t$ for $(s, a, t) \in \rightarrow$. The question of when two processes should be deemed to have consistent behaviour can be answered in many ways depending on context. One simple choice is simulation. A process $B=\left(S_{B}, i_{B}, L_{B}, \rightarrow_{B}\right)$ is said to simulate a process $A=\left(S_{A}, i_{A}, L_{A}, \rightarrow_{A}\right)$ if there exists a simulation relation $\mathcal{S} \subseteq S_{A} \times S_{B}$ containing $\left(i_{A}, i_{B}\right)$. The condition for the relation to be a simulation relation is the following:

$$
(s, t) \in \mathcal{S} \Rightarrow\left(\forall a, s^{\prime} .\left(s \stackrel{a}{\rightarrow} s^{\prime} \Rightarrow \exists t^{\prime} . t \stackrel{a}{\rightarrow} t^{\prime} \wedge\left(s^{\prime}, t^{\prime}\right) \in \mathcal{S}\right)\right)
$$

This can very easily be encoded as a game: starting at the start state of $A$, Refuter picks a transition. Verifier has to pick a transition from the start state of $B$ which has the same label. We now have a new pair of states, the targets of the chosen transitions, and the process repeats: again, Refuter chooses a transition from $A$ and Verifier has to match it. Play continues unless or until one player cannot go: either Refuter cannot choose a transition, because there are no transitions from his state, or Verifier cannot choose a transition because there is no transition from her state which matches the label on the transition chosen by Refuter. A player wins if the other player cannot move. If play continues for ever, Verifier wins. It is easy to show that in fact, Verifier has a winning 
strategy for this game exactly when there exists a simulation relation between the two processes; indeed, in a sense which can be made precise, a simulation relation is a winning strategy for Verifier. (As with the Skolem functions for $\Pi_{2}$ formulae, there may be more than one simulation relation between a given pair of processes.)

A curious and relevant fact about simulation is that even if $B$ simulates $A$ by simulation relation $\mathcal{S}$ and $A$ simulates $B$ by simulation relation $\mathcal{T}$, it does not follow that $A$ simulates $B$ by the reverse of $\mathcal{S}$, nor even that there must exist some relation which works as a simulation in both directions. This is the crucial difference between simulation equivalence and the stronger relation of bisimulation equivalence; see for example [2].

We will shortly define the semantics of QVT-R using a similar game, but first, we must consider an alternative approach.

\section{The translation from QVT Relations to QVT Core}

In an attempt to help readers and connect the several languages it defines, [5] defines the semantics of QVT Relations both directly, and by translation to QVT Core. Both specifications are informal (notwithstanding some minor use of logic e.g. in Appendix B). [5] does not specify what should happen in the case of conflicts between the two, nor does it explicitly argue for their consistency. Therefore any serious attempt to provide a formally-based semantics for QVT-R needs to take both methods into consideration. In this section, we consider the translation, with the aid of a very simple example QVT-R transformation. We then argue that, not only is what we believe to be the intended translation of this transformation not semantically equivalent, but also, the intended semantics of QVT Core appear to be such that it simply cannot express semantics equivalent to those of our simple QVT-R example. That is, even if our reading of the translation is incorrect, the problem remains: no translation can correctly reproduce the semantics of QVT-R. If the reader is convinced by the argument, it follows that the translation of QVT-R to QVT Core cannot contribute to an understanding of QVT-R.

Consider an extremely simple MOF metamodel which we will call SimplestMM. It defines one metaclass, called ModelElement, which is an instance of MOF's Class. It defines nothing else at all, so models which conform to this metamodel are simply collections (possibly empty) of instances of ModelElement. (Of course, in the usual object-oriented fashion, there is no obstacle to having several instances of ModelElement which are indistinguishable except by their identities.) We will refer to three models which conform to SimplestMM, having zero, one and two ModelElements respectively. We will imaginatively call them Zero, One and Two. Indeed, models conforming to SimplestMM can be identified in this way with natural numbers: a natural number completely determines such a model, and vice versa. 


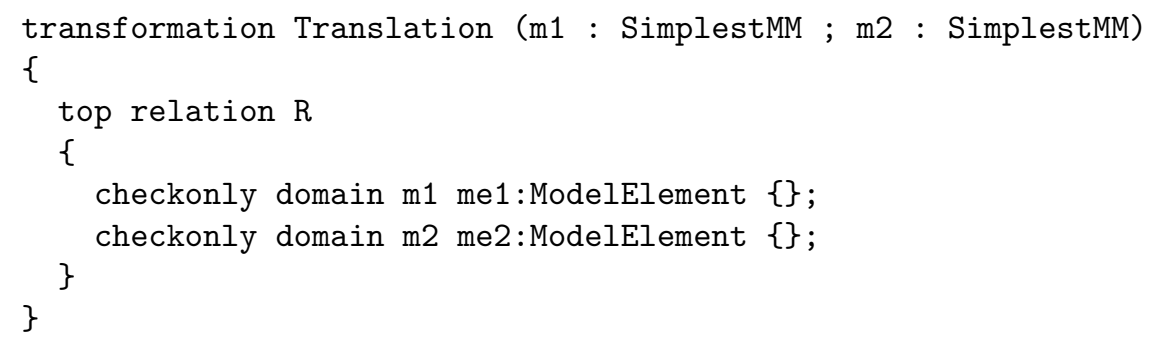

Figure 1: A very simple transformation

Next, consider a very simple QVT-R transformation between two models each of which conforms to SimplestMM. Figure 1 show the text of the transformation (we use ModelMorf syntax here).

Suppose that we use the QVT-R semantics to execute this transformation in the direction of $\mathrm{m2}$ (we will return to the issue of directionality of checkonly transformations below, in Section 4). When executed in the direction of $\mathrm{m} 2$, it should return true if and only if, for every valid binding of me1 there exists a valid binding of me2. There are no constraints beyond the type specification, so this is equivalent to: if model $\mathrm{m} 1$ is non-empty, then model $\mathrm{m} 2$ must also be non-empty. If model $\mathrm{m} 1$ is empty, then there is no constraint on model m2. Thus, when invoked on the six possible pairs of models from Zero, One and Two, the transformation should return false on the pairs (One,Zero) and (Two,Zero), otherwise true. Conversely, if we check in the direction of $\mathrm{m} 1$, the transformation returns false if $\mathrm{m} 1$ is empty and $\mathrm{m} 2$ is not, otherwise true. Reassuringly, ModelMorf gives exactly these results.

QVT-R works this way because its semantics are specified using logical "for all-there exists" formulae, without reference to a trace model or any other means of enforcing a permanent binding of one model element to another, such that a model element might be considered "used up". While [5] says that running a QVT-R transformation "implicitly" generates a trace model, the definition of the transformation does not rely upon its existence. It is simply assumed that an implementation will build a trace model, and use it, for example, to allow small changes to one model to be propagated to another without requiring all the computation involved in running a transformation to be redone. However, because the definition of QVT-R is independent of any trace model or its properties, there is no obstacle to the same model element being used more than once, which is why the transformation has the semantics discussed, rather than enforcing any more restrictive condition, such as that the two models have the same number of model elements. This helps to provide QVT-R the ability to express non-bijective transformations in the sense discussed in [10]; this ability in turn is essential to allow the expression of transformations between models which abstract away different things. The absolute requirement to be able to do this is most obvious when we consider a transformation between a fully-detailed 
model and an abstracted view onto it, where either the full model or the view may be updated (this is called the "view update problem" in databases). Even in transformations between models we might regard as equally detailed, though, it turns out that non-bijectiveness is essential. For example, in a realistic interpretation of a transformation between UML packages and RDBMS schemas, there are many schemas which are consistent with a given package, and many packages consistent with a given schema. See [10] for more discussion.

Now, taking [5] at face value, we expect to be able to translate this simple QVT-R transformation into a QVT Core transformation which has the same behaviour, and which, in particular, will return the same values when invoked on our simple models. The specification of the translation is not so clear that mistakes are impossible (e.g., possibly the multiple importing of the same metamodel is unnecessary), but this is what the author believes to be the intended translation:

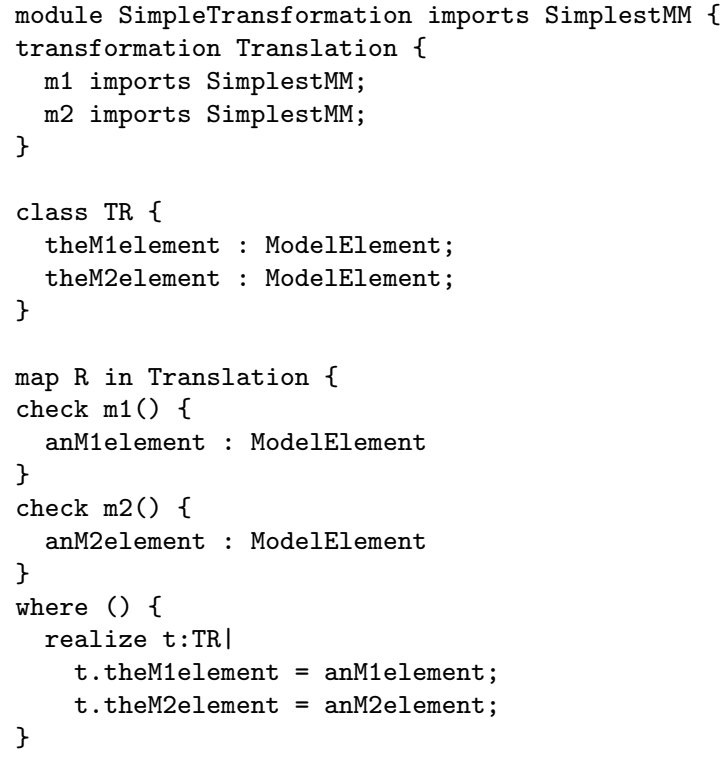

The effect of this QVT Core transformation is to construct for every model element in $\mathrm{m} 1$ an object of the trace class TR which connects this model element to a corresponding model element in m2. However, [5] says several times that in QVT Core, valid bindings must be unique. For example, p133 says:

There must be (exactly) one valid-binding of the bottom-middle pattern and (exactly) one valid binding of the bottom-domain pattern of a checked domain, for each valid combination of valid bindings of all bottom-domain-patterns of all domains not equal to the checked domain, and all these valid bindings must form a valid combination together with the valid bindings of all guard patterns of the mapping.

and this sentiment is then repeated in a logical notation. In executing the QVT Core version of our transformation on the models (Two,One), this condition 
would fail because, given the valid binding of the single ModelElement in One to variable me2, there would have to be two valid bindings to me1, one binding each of the ModelElements in Two. What is not so clear is whether this condition is intended to be satisfied if we run the example on (Two,Two): a literal reading would seen to suggest not, yet it seems impossible that QVT Core is intended to be unable to express the identity relation. The problem is where exactly the valid binding is supposed to be unique: in the model, or just in the mapping? That is, given a model element in $\mathrm{m} 2$, must there exist only one model element in $\mathrm{m} 1$ which could validly be linked to it, or is it, more plausibly, enough that there is only one model element which actually is linked to it by some trace object? Either way, though, (Two,One) will still fail.

Unfortunately no implementation of QVT Core seems to be available. Various sources refer to a pre-release of Compuware OptimalJ, but OptimalJ no longer exists. Therefore we cannot investigate what actual QVT Core tools do.

It is noteworthy, though, that this misapprehension that model elements, or at least patterns of them, must correspond one-to-one in order to make bidirectional transformations possible is pervasive: it appears even in the documentation for Medini QVT, which intends to be an implementation of QVT-R (see Medini QVT Guide, version 1.6, section QVT Relations Language, Bidirectionality). Indeed, Medini rejects many QVT-R transformations that are legal according to [5] and accepted by ModelMorf. For this reason we do not consider Medini any further.

Could we write a QVT Core transformation which did have the same behaviour as our simple QVT-R transformation? Unfortunately not. A moment's thought will show that the requirement that valid bindings correspond oneto-one (even if only in the constructed trace model) precludes any QVT Core transformation that could return true on both (One,Two) and (Two,One) but false on (One,Zero).

\section{Transformation direction}

The reader who is familiar with [10] may have noticed an inconsistency between the treatment of bidirectional transformations in that paper and the way we described checkonly transformations above. The framework in [10] is based on a direction-free notion of consistency: a transformation between sets of models $M$ and $N$ specifies, for any pair $(m, n) \in M \times N$, whether or not $m$ is consistent with $n$. In the above, however, our consistency check had a direction: checking Translation in the direction of $\mathrm{m} 2$ is not the same as checking it in the direction of $\mathrm{m} 1$ and indeed, can give different answers. When Translation is checked in the direction of $\mathrm{m} 1$ on the pair of models (Zero, One), it returns true, since there are no model elements on the left to be matched. When the same transformation is checked on the same pair of models in the other direction, it returns false.

The standard [5] is slightly ambivalent about whether a checkonly QVT$\mathrm{R}$ transformation has a direction. Compare p13, which talks about "checking two models for consistency" and implicitly contrasts execution for enforcement, 
which has a direction, with execution for checking, which implicitly does not, with the details of the QVT-R definition which clearly assume that checking has a direction. The resolution seems to be (p19, my emphasis):

A transformation can be executed in "checkonly" mode. In this mode, the transformation simply checks whether the relations hold in all directions, and reports errors when they do not.

That is, the notion of consistency intended by the QVT-R standard is given by conjunction: $\mathrm{m} 1$ is consistent with $\mathrm{m} 2$ according to transformation $R$ if and only if $R$ 's check evaluates to true in both directions.

In fact, ModelMorf requires a transformation execution to have a direction specified, even when it is checkonly: to find out what the final result of a checkonly transformation is, one has to manually run it in each direction and conjoin the results. Medini, by contrast, makes it impossible to run a transformation in checkonly mode: if you run a transformation in the direction of a domain which is marked enforce, there is no way to make the transformation engine return false if it finds that the models are inconsistent, rather than modifying the target model. These seems to be a misinterpretation of [5] and indeed is on the bug list. However, it is a superficial matter, because QVT-R is supposed to have "check then enforce" semantics: that is, it is not supposed to modify a model unless it is necessary to do so to enforce consistency. Therefore, given a QVT engine which was compliant with [5] except that it did not provide the ability to run transformations in checkonly mode, it would be easy to construct a fully compliant engine using a wrapper. The wrapper would save the target model, run the transformation, and compare the possibly modified target model with the original. If the target model had been modified, it would restore the original version and return false; otherwise, it would return true.

\section{A game-theoretic semantics for checkonly QVT- $\mathbf{R}$}

Given a set of metamodels, a set of models conforming to the metamodels, a transformation written in a simplified version of QVT-R, and a direction for checking, we will define a formal game which explains the meaning of the transformation in the following sense. The game is played between Verifier and Refuter. Refuter's aim in the game is to refute the claim that the check should succeed; Verifier's aim is to verify that the check should succeed. The semantics of QVT is then defined by saying that the check returns true if and only if Verifier has a winning strategy for the game. If this is not the case, then (since by Martin's standard theorem on Borel determinacy [4] the game we will define will be determined, that is, one or other player will have a winning strategy) Refuter will have a winning strategy, and this corresponds to the check returning false.

This approach has several advantages. Most importantly, it separates out the specification of what the answer should be from the issue of how to calculate 
the answer efficiently. Calculating a winning strategy is often much harder (in both informal, and formal complexity, senses) than checking that a given strategy is in fact a winning strategy. Indeed, it can be useful to calculate a strategy using heuristics or other unsound or unproved methods, and then use a separate process to check that it is winning: this is the game equivalent of a common practice in formal proof, the separation between the simple process of proof checking and the arbitrarily hard process of proof finding. Nevertheless, although this paper does not address the issue of how winning strategies can be calculated efficiently, it is worth noting that formulating the problem in this way makes accessible a wealth of other work on efficient calculation of winning strategies to similar games. ${ }^{4}$

We may also hope to be able to use the game to explain the meaning of particular transformations, or of the QVT-R language in general, to developers or anyone else who needs to understand it: similar approaches have proven successful in teaching logic and concurrency theory.

Finally, a game-theoretic approach is a helpful framework in which to consider the implications of minor variations in decisions about what the meaning of a QVT-R transformation should be, since many such differences arise as minor variations in the rules of the game.

In order to specify a two-player game of perfect information, we need, following Definition 2, to provide definitions of the positions, the legal moves, the way to determine which player should move from a given position, and the circumstances under which each player shall win.

We fix a set of models, where each $m_{i}$ conforms to a metamodel $M_{i}$, and a transformation definition given in a simplified version of QVT-R. Specifically, we consider that when and where clauses are only allowed to contain (conjunctions of lists of) relation invocations, not arbitrary OCL. We do not consider extension or overriding of transformations or relations. Further, our semantics is parametrised over a notion of pattern matching and relation-local constraint checking: in other words, we do not give semantics for these, but assume that an oracle is given to check the correctness, according to the relevant metamodel, of a player's allocation of values to variables, and local constraints such as identity of values between variables in different domains.

We will first define a game $G_{k}$ which corresponds to the evaluation of a QVT$\mathrm{R}$ checkonly transformation in the direction of one of its typed models, $m_{k}$. For ease of understanding we will explain the progress of the game informally first: Figure 2 defines the positions and the moves of the game more systematically. The player whose turn it is to move is encoded directly as the first element of the current position; Refuter moves from the initial position. At every stage, if it is a player's turn to move, but that player has no legal moves available, then the other player wins. As we shall discuss in a moment, we forbid infinite plays, so this completes the elements needed for a formal game definition, as listed in Definition 1.

\footnotetext{
${ }^{4}$ For the most complex games we consider here, such work is collated in the PGSolver project, http://www.tcs.ifi.lmu.de/pgsolver/. If we insist that the graph of relations should be a DAG, as discussed later in this section, simpler automata-based techniques suffice.
} 
To begin a play of game $G_{k}$, Refuter picks a top relation (call it $R$ ) and valid bindings for all patterns except that from $m_{k}$, and for any when variables (that is, variables which occur as arguments in relation invocations in the when clause of $R$ ). Notice that he is required to pick values which do indeed constitute valid bindings and satisfy relation-local constraints, as confirmed by the oracle mentioned earlier. Play moves to a position which we will notate (Verifier, $R, B, 1$ ), indicating that Verifier is to move, that the relation in play is $R$, that bindings in set $B$ have been fixed, and that only one of the players has yet played a part in this relation.

Verifier may now have a choice.

1. She may pick a valid binding for the as-yet-unbound variables from the $m_{k}$ domain (if any), such that the relation-local constraints such as identity of values of particular variables are satisfied according the oracle. Let the complete set of bindings, including those chosen by both players, be $B^{\prime}$. (If there are no more variables to bind, Verifier may still pick this and $B^{\prime}=B$.) In this case, play moves to a position which we will notate (Refuter, $R, B^{\prime}, 2$ ) indicating that Refuter is to move, that the relation in play is still $R$, that the bindings in set $B^{\prime}$ have been fixed, and that both players have now played their part in this relation.

2. Or, she can challenge one of the relation invocations in the when clause (if there are any), say $S$ (whose arguments, note, have already been bound by Refuter). Then play moves to $S$, and before finishing her turn, she must pick valid bindings for all patterns of $S$ except that from $m_{k}$, and for any when variables of $S$. Say that this gives a set of bindings $C$, in which the bindings of the root variables of all domains are those from $B$, and bindings of the other variables are those just chosen by Verifier. The new position is (Refuter, $S, C, 1$ ).

If Verifier chose 2., play proceeds just as it did from (Verifier, $R, B, 1$ ) except that, notice, the roles of the players have been reversed. It is now for Refuter to choose one of the two options above, in the new relation $S$.

If Verifier chose 1., Refuter's only option is to challenge one of the relation invocations in the where clause, say $T$ (whose arguments, note, are bound). (If there are none, he has no valid move, and Verifier wins this play.) Then play moves to $T$, and, before finishing his turn, Refuter must pick valid bindings for all patterns of $T$ except that from $m_{k}$, and for any when variables of $T$. Say that this gives a set of bindings $D$, in which the bindings of the root variables of all domains are those from $B^{\prime}$, and bindings of the other variables are those just chosen by Refuter. The new position is (Verifier, $T, D, 1$ ). Play now continues just as above.

The final thing we have to settle is what happens if play never reaches a position where one of the players has no legal moves available: who wins an infinite play? We may choose just to forbid this to happen, e.g., by insisting as a condition on QVT-R transformations that the graph in which nodes are relations and there is an edge from $R$ to $S$ if $R$ invokes $S$ in a where or when 


\begin{tabular}{|l|l|l|}
\hline Position & Next position & Notes \\
\hline Initial & Verif., $R, B, 1)$ & $\begin{array}{l}R \text { is any top relation; } B \text { comprises valid bindings for } \\
\text { all variables from domains other than } k, \text { and for any } \\
\text { when variables. } B \text { is required to satisfy domain-local } \\
\text { constraints on all domains other than } k .\end{array}$ \\
\hline$(P, R, B, 1)$ & $\left(\bar{P}, R, B^{\prime}, 2\right)$ & $\begin{array}{l}B^{\prime} \text { comprises } B \text { together with bindings for any remain- } \\
\text { ing variables. } B^{\prime} \text { is required to satisfy domain-local con- } \\
\text { straints on all domains. }\end{array}$ \\
\hline$(P, R, B, 1)$ & $(\bar{P}, S, C, 1)$ & $\begin{array}{l}S \text { is any relation invocation from the when clause of } \\
R ; C \text { comprises } B \text { 's bindings for the root variables of } \\
\text { patterns in } S, \text { together with valid bindings for all vari- } \\
\text { ables from domains other than } k \text { in } S, \text { and for any when } \\
\text { variables of } S . C \text { is required to satisfy domain-local } \\
\text { constraints on all domains other than } k .\end{array}$ \\
\hline$(P, R, B, 2)$ & $(\bar{P}, T, D, 1)$ & $\begin{array}{l}T \text { is any relation invocation from the where clause of } \\
R ; D \text { comprises } B \text { 's bindings for the root variables of } \\
\text { patterns in } T, \text { together with valid bindings for all vari- } \\
\text { ables from domains other than } k \text { in } T, \text { and for any when } \\
\text { variables of } T . D \text { is required to satisfy domain-local con- } \\
\text { straints on all domains other than } k .\end{array}$ \\
\hline
\end{tabular}

Figure 2: Summary of the legal positions and moves of the game $G_{k}$ : note that the first element of the Position says who picks the next move, and that we write $\bar{P}$ for the player other than $P$, i.e. $\overline{\text { Refuter }}=$ Verifier and vice versa. Recall that bindings are always required to satisfy relevant metamodel and relation-local constraints.

clause, should be acyclic. There is probably ${ }^{5}$ a reasonable alternative that achieves sensible behaviour by allowing the winner of an infinite play to be determined by whether the outermost clause which is visited infinitely often is a where clause or a when clause: but this requires further investigation. Note that [5] has nothing to say about this situation: it corresponds to infinite regress of its definitions. For now, we will forbid infinite plays, and declare any QVT-R transformation with a cyclic when-where graph to be ill-formed.

\subsection{Discussion of the treatment of when clauses}

Most of the above game definition is immediate from [5], but the treatment of when clauses requires discussion. From Chapter 7, ([5], p14): "The when clause specifies the conditions under which the relationship needs to hold, so the relation ClassToTable needs to hold only when the PackageToSchema relation holds between the package containing the class and the schema containing the table."

\footnotetext{
${ }^{5}$ by thinking from first principles about cases in which a play goes through a when (rsp. where) clause infinitely often, but only finitely often through where (rsp. when) clauses; or by intriguing analogy with $\mu$ calculus model-checking
} 
The naive way to interpret this would have been to say that both Refuter and Verifier choose their values, and then, if it turns out that the when clause is not satisfied given their choices, Verifier wins this play. This interpretation is not useful, however, as it often gives Verifier a way to construct a winning strategy which does not tell us anything interesting about the relationship between the models. When challenged by Refuter to pick a value for her domain, all she would need to do would be to pick a binding such that the when clause was not satisfied. In the case discussed by [5], whenever Refuter challenged with a class, she would reply with any table from a schema not corresponding to the package of his class, the when clause would not be satisfied, and she would win.

So the sense in which a when clause is a precondition must be more subtle than this. In programming, giving a function a precondition makes it easier for the function satisfy its specification, but here the idea is rather to restrict Verifier's choices: if Refuter chooses a class $C$ in package $P$, Verifier is bound to reply not with any table, but specifically with a table $T$ which is in a/the schema that corresponds to package $P$.

The sense in which this is a precondition is that the facts about what packages correspond to what schemas are supposed to have been pre-computed: but the order of computation of facts is not something we need to concern ourselves with here, since we are not interested in efficiency but only in meaning.

In trying to settle whether we really mean "a schema" or "the schema" in the paragraph above, we refer again to Appendix B of [5]. The problem is that this is not a complete definition. E.g., in order to use it to interpret ClassToTable, we already need to be able to determine whether, for given values of a package $p$ and schema $s$, the when clause when \{ PackageToSchema $(\mathrm{p}, \mathrm{s})$ \} holds. Informally it seems that people who write about QVT have two different interpretations of this, perhaps not always realising that they are different:

1. the purely relational: the pair $(p, s)$ is any member of the relation expressed in PackageToSchema, when it is interpreted using the very same text which we are now trying to interpret

2. the operational: the program which is checking the transformation is assumed to have looked at PackageToSchema already and chosen a schema to correspond to package $\mathrm{p}$ (recording that choice using a trace object). According to this view, we only have to consider $(p, s)$ if $s$ is the very schema which was chosen on this run of the checking program.

To see the difference, imagine that there are two schemas, s1 and s2, either of which could be chosen as a match for $p$ in PackageToSchema. In the first interpretation, both possibilities have to be checked when ClassToTable is interpreted; in the second, only whichever one was actually used.

In our main game definition, we have taken the purely relational view, since we can do so while remaining compatible with the definitions in [5], whereas as we have seen in the SimplestMM example - which, recall, had no when or where clauses and whose semantics were therefore defined unambiguously by Appendix $\mathrm{B}$ - the idea that there should be a one-to-one correspondence between valid 
bindings is incompatible with Appendix B; but we will shortly consider a variant of the game which brings us closer to the latter view.

\section{Examples and comparison with QVT-R im- plementations}

In this section we use a family of simple examples to illustrate the game-based semantics. We go on to compare the semantics it implements with what is implemented by ModelMorf.

\subsection{A family of examples}

We use a metamodel which defines just one metaclass, ABoolean, which defines just one boolean value. We denote using $T, F$, the simplest two models conforming to this metamodel, each of which defines one ABoolean with value true, false respectively. These models and the metamodel are shown (in a form suitable for ModelMorf) in Figure 3. For convenience we will refer to the single model element in each model as $t t, f f$ respectively.

To illustrate the effects of when and where clauses, we will consider the very simple transformations shown in Figures 4 to 7 , which all use the same two basic relation definitions but differ in how these are put together using when and where invocations.

\subsection{The examples in our semantics}

Running each of our example transformations, on each of the four possible pairs of models, in each of the two directions, yields 32 examples. In each case, the semantics must return true or false: true if the two models are considered consistent according to the transformation in the considered direction, false otherwise. In our game-based semantics, the result is, by definition, true if Verifier has a winning strategy for the game, otherwise false. That is, a demonstration that the semantics gives result true on a particular problem is a winning strategy for Verifier no the game; a demonstration that the semantics gives result false is a winning strategy for Refuter on the game.

First let us see how this works for PwhereQ run on the pair of models $(T, T)$ in the direction of $\mathrm{m} 2$. Unsurprisingly the result of this checkonly transformation, according to both our semantics and ModelMorf, is true, which we will demonstrate by exhibiting a winning strategy for Verifier.

Play begins, as always, at the Initial position from which Refuter is to move. Reading off from Figure 2, he has to choose a top relation, and there is only one, viz. SameValue. He also has to choose valid bindings from domains other than $\mathrm{m} 2$; that is, he must choose an ABoolean $s 1$ and its value $i$. Again, he has only one option. There are no when variables so he is done; the only possible play so far is 
Metamodel BoolMM.xml

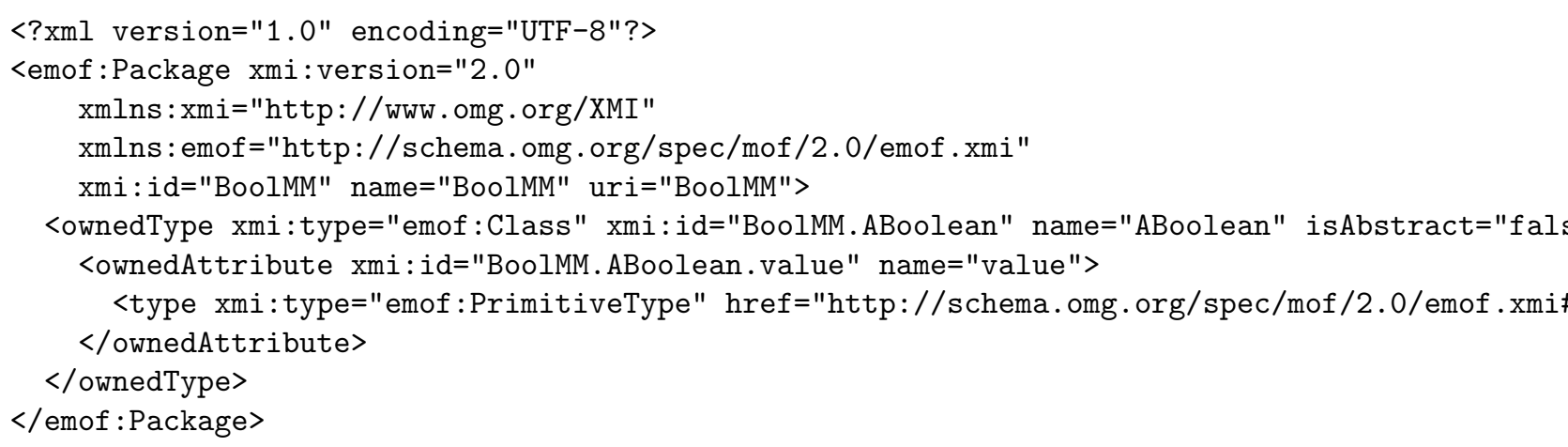

Model true.xml

<?xml version="1.0" encoding="ASCII"?>

<xmi:XMI xmi:version="2.0" xmlns:xmi="http://www.omg.org/XMI" xmlns:BoolMM="BoolMM"> <BoolMM:ABoolean xmi:id="BoolMM.ABoolean.1" value="true"/> $</ \mathrm{xmi}: \mathrm{XMI}>$

Model false.xml

<?xml version="1.0" encoding="ASCII"?>

<xmi:XMI xmi:version="2.0" xmlns:xmi="http://www.omg.org/XMI" xmlns:BoolMM="BoolMM"> <BoolMM:ABoolean xmi:id="BoolMM.ABoolean.1" value="false"/> $</ x m i$ :XMI $>$

Figure 3: The examples' metamodel and two models 


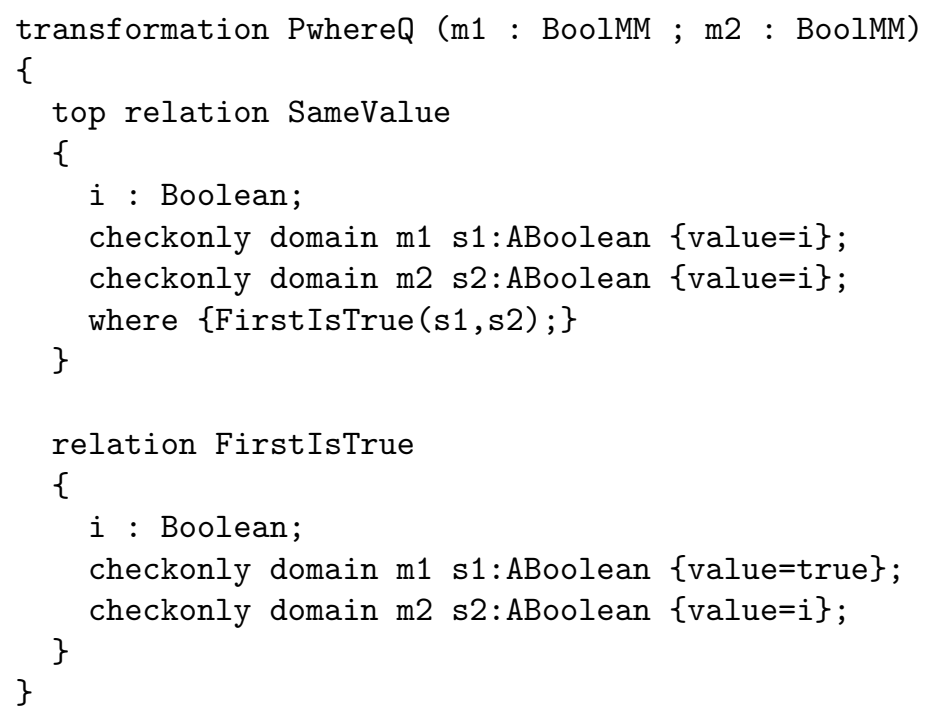

Figure 4: Transformation PwhereQ

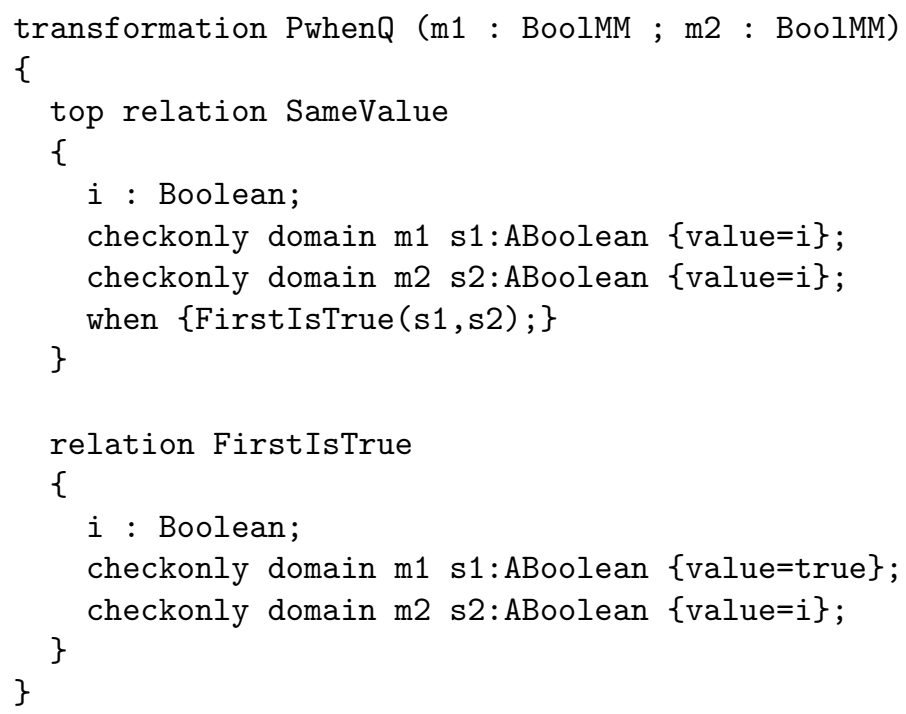

Figure 5: Transformation PwhenQ 


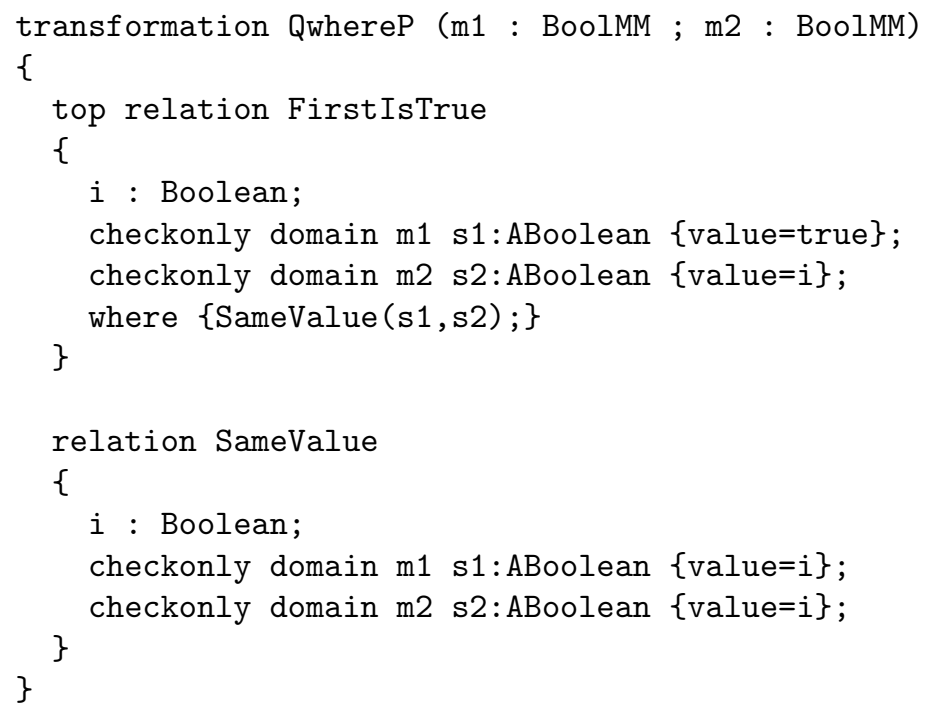

Figure 6: Transformation QwhereP

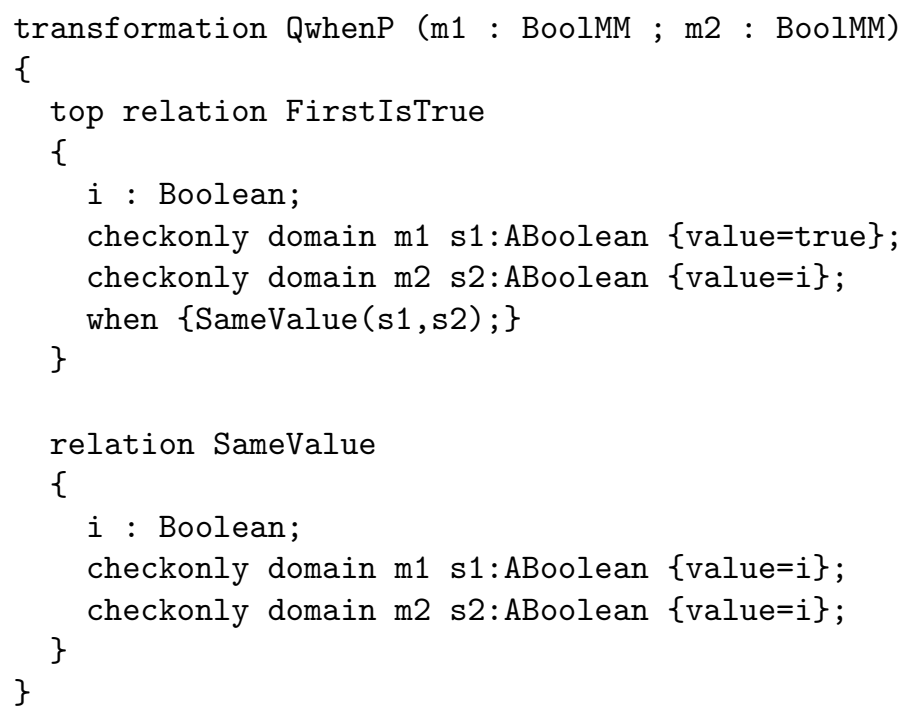

Figure 7: Transformation QwhenP 


\section{Initial, (Verifier, SameValue, $\{s 1 \mapsto t t, i \mapsto$ true $\}, 1)$}

What are the legal moves from this position? There is no when clause so the legal move must come from line 2 of Figure 2: Verifier has to extend the set of bindings to include a binding for $s 2$. Notice that she does not choose a binding for $i$, even though it does appear in the $\mathrm{m} 2$ domain clause in the current relation, because $i$ has already been bound by Refuter's choice. She has only one choice for $s 2$, so there is only one legal move, and this must be the move that our strategy prescribes. Play continues from the new position

$$
\text { (Refuter, SameValue, }\{s 1 \mapsto t t, s 2 \mapsto t t, i \mapsto \text { true }\}, 2)
$$

We see from the final line of Figure 2 that Refuter now needs to pick a relation invocation from the where clause of the current relation - that is, the invocation of FirstIsTrue. The set of bindings in the new position includes the already existing bindings for the root variables of patterns in FirstIsTrue, that is, the bindings $\{s 1 \mapsto t t, s 2 \mapsto t t\}$ are retained. Notice, however, that these are the only bindings that are retained: the binding of $i$ is discarded at this point. Now Refuter chooses bindings for any variables other than the root in domain $\mathrm{m} 1$, and for any when variables - but there are none, so we simply check that Refuter's trivial "choice" constitutes a valid binding - which it does, since the value in $t t$ is true - and we are done. The new position is

$$
\text { (Verifier, FirstIsTrue, }\{s 1 \mapsto t t, s 2 \mapsto t t\}, 1 \text { ) }
$$

Verifier needs to add a binding for $i$; in our scenario this binding must be to true, although in fact it's unconstrained, giving new position

$$
\text { (Refuter, FirstIsTrue, }\{s 1 \mapsto t t, s 2 \mapsto t t, i \mapsto \text { true }\}, 2)
$$

At this point, Refuter has no legal move, since there is no where clause. Therefore Verifier wins this play. Since at no stage did Refuter have any alternative choices that might have proved more successful for him, we can immediately say that Verifier's winning strategy is

$$
\begin{gathered}
S=\quad \text { (Verifier, SameValue, }\{s 1 \mapsto t t, i \mapsto \text { true }\}, 1) \mapsto \\
\text { (Refuter, SameValue, }\{s 1 \mapsto t t, s 2 \mapsto t t, i \mapsto \text { true }\}, 2), \\
\quad(\text { Verifier, FirstIsTrue, }\{s 1 \mapsto t t, s 2 \mapsto t t\}, 1) \mapsto \\
\text { (Refuter, FirstIsTrue, }\{s 1 \mapsto t t, s 2 \mapsto t t, i \mapsto \text { true }\}, 2)\}
\end{gathered}
$$

In general, of course, a tool might have to search for a winning strategy, and, whilst a depth-first exploration of play will certainly work for games with no infinite plays, much more efficient searches might be possible. The advantage of the game-based semantics, from the point of view of understandability, is that it does not matter how the strategy is found. Given a strategy, it is easy (both 


\begin{tabular}{l|cccc}
$\leftarrow$ & $(\mathrm{T}, \mathrm{T})$ & $(\mathrm{T}, \mathrm{F})$ & $(\mathrm{F}, \mathrm{T})$ & $(\mathrm{F}, \mathrm{F})$ \\
\hline PwhereQ & $\mathrm{V}$ & $\mathrm{R}$ & $\mathrm{R}$ & $\mathrm{R}$ \\
PwhenQ & $\mathrm{V}$ & $\mathrm{R}$ & $\mathrm{V}$ & $\mathrm{V}$ \\
QwhereP & $\mathrm{V}$ & $\mathrm{R}$ & $\mathrm{R}$ & $\mathrm{R}$ \\
QwhenP & $\mathrm{V}$ & $\mathrm{V}$ & $\mathrm{V}$ & $\mathrm{R}$
\end{tabular}

Figure 8: Results in the direction of $\mathrm{m} 1$

\begin{tabular}{l|cccc}
$\rightarrow$ & $(\mathrm{T}, \mathrm{T})$ & $(\mathrm{T}, \mathrm{F})$ & $(\mathrm{F}, \mathrm{T})$ & $(\mathrm{F}, \mathrm{F})$ \\
\hline PwhereQ & $\mathrm{V}$ & $\mathrm{R}$ & $\mathrm{R}$ & $\mathrm{V}$ \\
PwhenQ & $\mathrm{V}$ & $\mathrm{R}$ & $\mathrm{R}$ & $\mathrm{V}$ \\
QwhereP & $\mathrm{V}$ & $\mathrm{R}$ & $\mathrm{V}$ & $\mathrm{V}$ \\
QwhenP & $\mathrm{V}$ & $\mathrm{V}$ & $\mathrm{V}$ & $\mathrm{V}$
\end{tabular}

Figure 9: Results in the direction of $\mathrm{m} 2$

cognitively and computationally) to check that it is a winning strategy, which is what is required.

Next let us consider an example containing a when clause: PwhenQ run on $(\mathrm{F}, \mathrm{T})$ in the direction of $\mathrm{m} 2$. This proves even simpler. Play must begin with Refuter moving from Initial to (Verifier, SameValue, $\{s 1 \mapsto f f, s 2 \mapsto t t, i \mapsto$ false, 1$)$. From here, Verifier has no legal move. She cannot use line 2 of Figure 2, because (even though she has no values to bind) she would be required to ensure the satisfaction of the domain-local constraint that the value in $t t$ was false, which of course she cannot do. She cannot use line 3 , challenging the when clause, because she would be required to ensure the satisfaction of the domain-local constraint that the value in $f f$ was true, which again she cannot do. Therefore, since it is Verifier's turn to move and she cannot do so, Refuter wins the play. Refuter has a winning strategy which consists simply of moving from Initial to the only possible position.

Now let us go on to summarise the results on the whole family of examples. Figure 8 shows the results when the transformations are checked in the direction of $\mathrm{m} 1$. Figure 9 shows the results when the transformations are checked in the direction of $\mathrm{m} 2$.

\subsection{Comparison with ModelMorf}

Running the set of examples discussed on this section in ModelMorf yields agreement in 31 of the 32 cases. The exception is PwhenQ run on $(\mathrm{F}, \mathrm{T})$ in the direction of $\mathrm{m} 2$, where our semantics gives false in contrast to ModelMorf's true. On the author's reporting this to Sreedhar Reddy, he confirmed that false is the correct answer[6], so that this was a bug in ModelMorf (which may have been corrected in the latest, but currently unavailable, version).

We have already demonstrated the application of the game-based semantics in this case (see above). Expanding the definitions in [5] shows that the result should be true iff 


$$
\text { FirstIsTrue }(\mathrm{s} 1, \mathrm{~s} 2) \Rightarrow \mathrm{s} 1 . \mathrm{value}=\mathrm{s} 2 \text {. value }
$$

To evaluate this we need to know what FirstIsTrue $(\mathrm{s} 1, \mathrm{~s} 2)$ means. Unfortunately, [5] does not discuss how to evaluate such an expression - it only defines relations without parameters. That definition is of the form "for all valid bindings... there exists a valid binding such that... conditions". A sensible interpretation of the parameterised case would therefore seem to be that for all valid completions of $\mathrm{s} 1$ to a valid binding (recalling that a valid binding has to satisfy domain-local constraints), there should exist a valid completion of s2 to a valid binding, such that the conditions hold on those bindings. In other words, specifying values for certain variables restricts the scope of the bindings that need to be considered in the definition; some decisions have already been made. This seems consistent with the intention of [5].

In this case, there is no way to complete $\mathrm{s} 1$ to a valid binding, since the definition of FirstIsTrue insists that the value in s1 should be true, which it is not. Therefore, the universally quantified formula is vacuously true, explaining why the final result of the checking transformation is true, and consistent with the answer produced by our game-based semantics.

It is encouraging that, on all the cases we have explored with the exception on the one case which has been confirmed to be a bug in ModelMorf, our semantics gives the same answers as ModelMorf. This suggests that our semantics has resolved ambiguities in [5] in a way compatible with the way the authors of QVT intended (since several of the same people are involved in both the document and the tool). Thus the game-based semantics may be useful as a way of explaining the intended meaning of QVT-R transformations, and perhaps of exploring further possibilities such as debugging tools, without needing to argue for a different meaning of transformations.

\section{Duality}

An intriguing aspect of the QVT-R language is that it seems that when and where clauses are in a certain sense dual. As far as we are aware, however, there are no results on this subject in the literature. In this section we show how the game-based semantics can help to access provably correct statements along these lines, and we give an example.

Inspection of the definition of the moves of the game $G_{k}$ as shown in Figure 2 shows that it is only in moving from Initial that we need to specify a player (Verifier or Refuter) by name: in every other kind of move, we simply swap or preserve the player, without needing to know whether we started with a Verifier or a Refuter position. (We may also note that the Initial move is also the only place in the game definition where it matters whether a relation is or is not designated a top relation.) Moreover, since our game definition and strategies are memoryless, it makes sense to talk about a winning strategy from a given position, not only from the Initial position. Immediate from this is the following observation: 
Lemma 1. Fix a game $G_{k}$. Let $P$ be either Verifier or Refuter, and let $\bar{P}$ be the other player. Independently, let $A$ be either Verifier or Refuter, and let $\bar{A}$ be the other player, and let $i$ be either 1 or 2 . Then $P$ has a winning strategy starting from position $(A, Q, B, i)$ iff $\bar{P}$ has a winning strategy starting from position $(\bar{A}, Q, B, i)$.

Proof. The same strategy will work in both cases. More precisely, suppose we are given a winning strategy for $P$ from $(A, Q, B, i)$, that is, a partial map $S$ from positions to positions which satisfies the conditions to be a winning strategy from $(A, Q, B, i)$. Construct a new partial map $\bar{S}$ from positions to position by replacing $A$ by $\bar{A}$ and vice versa wherever they occur. By duality of the game rules, $\bar{S}$ is a winning strategy for $\bar{P}$ from $(\bar{A}, Q, B, i)$.

This lemma can be used to compose what we know about different parts of a game graph. Here is a deliberately simple example:

Proposition 1. Fix a set $m_{i}$ of models. Consider two transformations, $T$ and $T^{\prime}$, which differ only in their definitions of one relation, their unique top relation, which is not invoked by any other relation; let $T$ 's unique top relation be $P$ while $T^{\prime}$ 's is $P^{\prime}$. In $P$, there is a when clause that simply invokes relation $Q$ with arguments $s_{i}$, and there is no where clause. In $P^{\prime}$, there is a where clause that simply invokes $Q$ with the same arguments, and there is no when clause. Moreover, $P, P^{\prime}$ and $Q$ satisfy the following conditions with respect to the models $m_{i}$ :

1. in each of relations $P, P^{\prime}$, there is a unique choice of valid bindings for the variables in domains other than $m_{k}$ (satisfying the domain-local constraints of domains other than $m_{k}$ ) and for the arguments to $Q$, and these bindings assign the same values to the arguments of $Q$;

2. in $P$ there is no valid binding of the variables in domain $m_{k}$ that, together with the unique choice of valid binding for the other variables, also satisfies the domain-local constraints on $m_{k}$;

3. in $P^{\prime}$ there is a valid binding of the variables in domain $m_{k}$ that, together with the unique choice of valid binding for the other variables, also satisfies the domain-local constraints on $m_{k}$;

4. in $Q$ there is a unique choice of valid bindings for the variables in domains other than $m_{k}$ (satisfying the domain-local constraints of domains other than $m_{k}$ ).

Then the checkonly transformation $T$ run on the set $m_{i}$ of models in the direction of $m_{k}$ returns true iff the transformation $T^{\prime}$ run on the same set of models in the same direction returns false.

Proof. The effect of the properties insisted on is to ensure that, from Initial, play in the $T$ game in can only proceed to (Refuter, $Q, B^{\prime}, 1$ ), where $B^{\prime}$ is the unique possible set of bindings, and similarly play in the $T^{\prime}$ game can only proceed to 
(Verifier, $\left.Q, B^{\prime}, 1\right)$. From this point, Lemma 1 gives the result, since the reachable portions of the game graph are indistinguishable from those points.

Notice that although we have imposed very stringent conditions on the relations $P, P^{\prime}, Q$, here, it is permitted that $Q$ invoke other relations that can be arbitrarily complex. For a concrete example, take $T$ to be PwhenQ from Figure 5, run in direction $\mathrm{m} 2$, the models to be $(F, T)$, and $T^{\prime}$ to be a variant that replaces the when clause by a where clause and imposes no constraints in the top relation.

Very informally, we may say that this result captures the observation that the transformation " $P$ where $Q$ " is equivalent to the negation of " $\bar{P}$ when $Q$ " where $P$ and $\bar{P}$ are opposites in a suitable sense such as the one imposed by the conditions above. Of course many variants on this result are possible: we have presented a particularly simple case for purposes of exposition. For example, it is not necessary to insist that there should be a unique set of valid bindings in each place where we did so, provided that care is taken to insist that choices and the players who choose them match up appropriately. Nevertheless, the need to take care over these aspects intuitively explains why no really general duality result seems to hold. More practical experience with QVT-R will be required to see what what examples might actually be interesting, for purposes of efficient implementation or otherwise.

\section{Variants of the game}

One of the advantages of the game-based approach to defining semantics is that it provides an intuitive means of examining the design decisions which have been made in choosing one semantics over another. In this section, we examine some alternatives.

\subsection{Non-directional variant}

Let $G$ be the variant of $G_{k}$ in which, instead of a direction being defined as part of the game definition, Refuter is allowed to choose a direction ("once and for all") at the beginning of the play. Clearly, Verifier has a winning strategy for $G$ if and only if she has a winning strategy for every $G_{k}$. This is the way of constructing a non-directional consistency definition from directional checks that is specified in [5]. However, note that it is not automatic that there should be any simple relationship between the various winning strategies; hence, there may not be any usable multi-directional trace relationship between the bindings in different models. In order to explain this, we need a digression on trace objects in QVT-R and how they relate to the game-based semantics.

\subsection{Trace objects and the game-based semantics}

Because QVT-R, as already stated, does not depend on the definition of any trace objects, the precise nature of the trace objects which are assumed to exist 
is not specified. Informally, it is clear that a trace object is supposed to link model elements which correspond in some sense expressed in the transformation. Since QVT-R can express non-bijective transformations, this linkage need not be one-to-one. Which objects correspond depends on which relation one looks at; there is nothing to stop a transformation involving many relations whose clauses impose different correspondences. Often, however, each model element will be relevant to - specifically, a possible value for a root variable in - exactly one top relation, so that the top relations partition the model elements. For example, packages and schemas are relevant to PackageToSchema, while classes and tables are relevant to ClassToTable, in the classic example from [5]. Consider the game $G_{k}$ defined using such a transformation. If Verifier has a winning strategy, she must be able to answer any initial challenge by Refuter, and after she has done so, the position contains a tuple of values for the root variables of all domains (and possibly other bindings as well). We may choose to define a link from the tuple of values for root variables of domains other than $m_{k}$ to the value for the root variable of $m_{k}$, and call it a trace object. Note, however, that it might not be Verifier who chooses the value for the $m_{k}$ root variable (if, for example, this variable happens to be a when variable in the relation) and therefore, even if our Verifier winning strategy is deterministic, it may not give rise to a set of trace objects that can be read as a function.

Supposing that we are happy with this definition of what it is to be a trace object, a winning strategy for Verifier then gives rise to a set of trace objects for the transformation. Of course, since the game is directional, there is an inherent direction to this set of trace objects: from tuples of model elements in models other than $m_{k}$, towards model elements in $m_{k}$. Note in passing that it is in principle possible for a transformation engine to determine that a winning strategy for Verifier exists without actually calculating one. Therefore it is not inevitable that evaluating a checkonly transformation on a set of models (and returning true or false) involves generating a set of trace objects.

Now let us explain the non-existence of bidirectional trace objects in the non-directional game $G$ using an example derived from one in [2].

Figure 10 illustrates two models which conform to the obvious metamodel MM: a model may include multiple Containers, each of which references one Inter, each of which may reference multiple Things, each of which has a value. The following QVT-R transformation evaluates to true on the models shown, in both directions (both according to [5], and according to ModelMorf). Indeed, Verifier has a winning strategy for $G$ : the only interesting choice she has to make is in $G_{2}$, where she has to be sure to reply with a2 (and i2), not a1 (and i1), if Refuter challenges in ContainersMatch by binding xa to c1 (and xi to inter1).

transformation Sim (m1 : MM ; m2 : MM)

\{

top relation ContainersMatch

\{

inter1,inter2 : MM: : Inter;

checkonly domain $\mathrm{m} 1 \mathrm{c} 1$ :Container inter = inter1\}; 

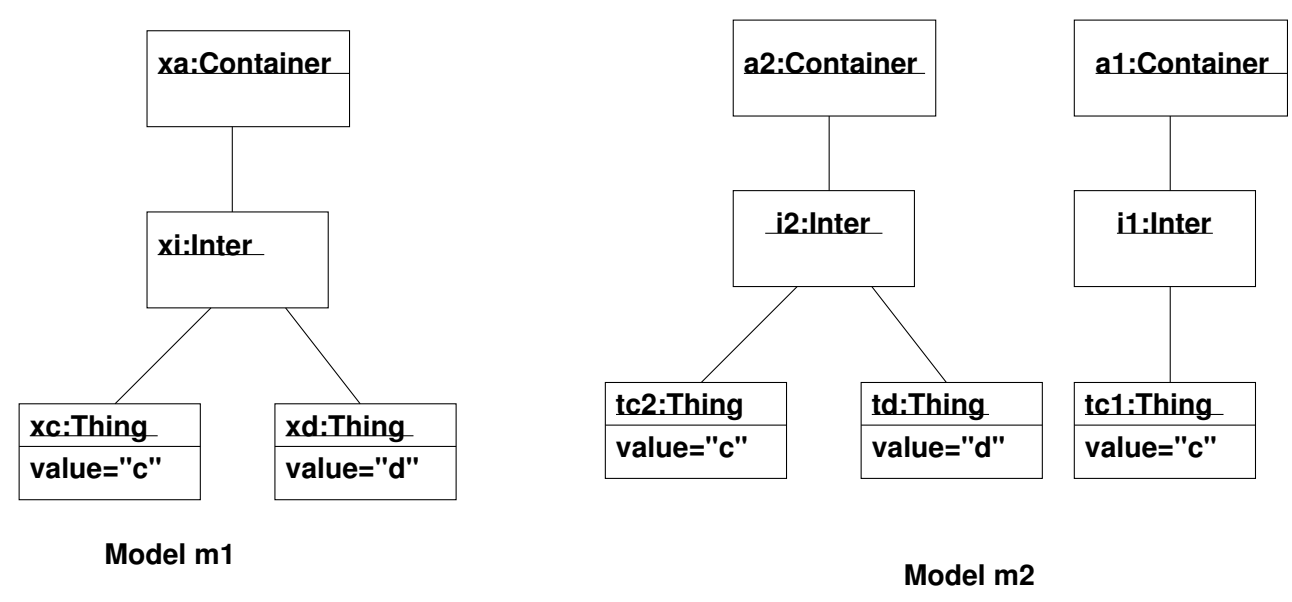

Figure 10: $\mathrm{m} 1$ and $\mathrm{m} 2$ are (two-way) consistent according to QVT-R transformation Sim, but no set of bi-directional trace objects can link them

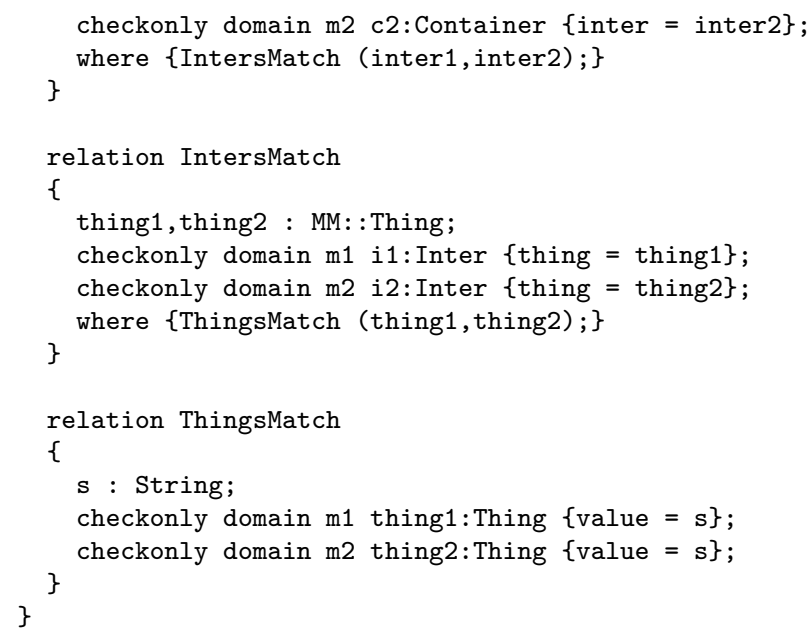

Now, in the $\mathrm{m} 1$ direction the constructed trace will take a1 to xa, etc.; there is nothing else it can do. Yet in the $\mathrm{m} 2$ direction, a trace object which took xa to a1 would be erroneous. Thus there can be no single set of trace objects whose links can be read in either direction, which could capture the correctness of this QVT-R transformation.

\subsection{Model-switching variant}

Let $G^{\prime}$ be the variant of $G$ in which, instead of the first player to move in a new relation being constrained to pick a valid binding everywhere except in the once-and-for-all designated target model $m_{k}$, the player is permitted to pick 
valid bindings for all but any one domain, making a new choice of which domain to leave out every time. This is a different way to define a non-directional variant of the game. The modification to the game rules is analogous to the difference, in concurrency theory, between a game which defines bisimulation equivalence and that which defines simulation equivalence. Formally, looking at the positions and moves in Figure 2, we would simply need to modify the positions of the form $(\ldots ., 1)$, by adding an additional integer element specifying in which domain the challenge is to be answered, that is, which domain may not have all its bindings chosen yet. The legal moves that result in such positions would have to specify that the player making the move has to choose which domain that shall be.

Having made this modification to the game, what is the effect semantically? A winning strategy for Verifier in the game $G^{\prime}$ can still be regarded as determining a set of trace objects, by reading off the tuples of model elements that occur as values for root variables of domains in positions that form part of her winning strategy. In this sense bidirectional trace objects will exist in $G^{\prime}$. However, the price may be that Verifier too seldom has a winning strategy: this corresponds to the observation that for many practical purposes in concurrency, bisimulation equivalence proves to be too strong an equivalence. Certainly in the example above, it will be Refuter who has a winning strategy for $G^{\prime}$ : he will first challenge in $\mathrm{m} 2$ with $\mathrm{a} 1$, and later switch to $\mathrm{m} 1$ where he leads play to the "d" which cannot be matched starting from a1 in $\mathrm{m} 2$.

\subsection{Trace-based variant}

Let $G^{T}$ be the variant of $G$ in which, as play proceeds, we build a global auxiliary structure which records, for each relation, what choices of valid binding have been made by the players (for example, "Package $P$ was matched with schema $S$ "). It is an error if subsequent moves in a play try to choose differently (and we might consider a multi-directional subvariant in which either matching $P$ with $S^{\prime}$ or matching $S$ with $P^{\prime}$ was an error, along with uni-directional subvariants in which only one of those would be an error). The player to complete such an erroneous binding would immediately lose. Otherwise, play would be exactly as in $G_{k}$, except that it loops: if Refuter cannot go, he can "restart", choose a new top relation and play again, but the old auxiliary structure is retained. If play passes through infinitely many restarts, Verifier wins. This game would impose one-to-one constraints on valid bindings, and construct well-defined trace objects, at the expense of having a semantics incompatible with [5] and having curtailed expressivity.

\section{Conclusions}

We have presented a game-theoretic semantics of QVT-R checkonly transformations, based on the direct semantics in [5]; we justified our choice to ignore the translation to QVT Core by pointing out a fundamental incompatibility between the two languages. We have briefly discussed variants of the game, 
demonstrating in the process that bi-directional trace objects may not exist.

\section{References}

[1] Miguel Garcia. Formalization of QVT-Relations: OCL-based Static Semantics and Alloy-based Validation. In Proceedings of the Second Workshop on MDSD Today, pages 21-30, October 2008.

[2] R.J. van Glabbeek. The linear time - branching time spectrum I; the semantics of concrete, sequential processes. In J.A. Bergstra, A. Ponse, and S.A. Smolka, editors, Handbook of Process Algebra, chapter 1, pages 3-99. Elsevier, 2001.

[3] Joel Greenyer and Ekkart Kindler. Reconciling TGGs with QVT. In Proceedings of 10th International Conference on Model Driven Engineering Languages and Systems, MODELS 2007, volume 4735 of Lecture Notes in Computer Science, pages 16-30. Springer, 2007.

[4] Donald A. Martin. Borel determinacy. Annals of Mathematics. Second series, 102(2):363-371, 1975.

[5] OMG. MOF2.0 query/view/transformation (QVT) version 1.0. OMG document formal/2008-04-03, 2008. available from www.omg.org.

[6] Sreedhar Reddy. personal communication, 26th November 2009.

[7] Raphael Romeikat, Stephan Roser, Pascal Müllender, and Bernhard Bauer. Translation of QVT relations into QVT operational mappings. In ICMT '08: Proceedings of the 1st international conference on Theory and Practice of Model Transformations, pages 137-151, Berlin, Heidelberg, 2008. Springer-Verlag.

[8] Perdita Stevens. A landscape of bidirectional model transformations. In Generative and Transformational Techniques in Software Engineering II, volume 5235 of Lecture Notes in Computer Science, pages 408-424. Springer, 2008.

[9] Perdita Stevens. Towards an algebraic theory of bidirectional transformations. In Proceedings of the International Conference on Graph Transformations, ICGT'08, volume 5214 of Lecture Notes in Computer Science, pages 1-17. Springer, September 2008. invited paper.

[10] Perdita Stevens. Bidirectional model transformations in QVT: Semantic issues and open questions. Journal of Software and Systems Modeling (SoSyM), 2009. to appear.

[11] Perdita Stevens. A simple game-theoretic approach to checkonly QVT Relations. In Proceedings of the International Conference on Model Transformations, ICMT'09, Lecture Notes in Computer Science. Springer, June 2009 . 
[12] Colin Stirling. Bisimulation, model checking and other games. In Notes for Mathfit Instructural Meeting on Games and Computation, 1997. Available from http://homepages.inf.ed.ac.uk/cps/mathfit.ps.

[13] Jennifer Tenzer and Perdita Stevens. GUIDE: Games with UML for interactive design exploration. Journal of Knowledge Based Systems, 20(7), October 2007. 